\title{
Three-Loop Color-Kinematics Duality: A 24-Dimensional Solution Space Induced by New Generalized Gauge Transformations
}

\author{
Guanda Lin, ${ }^{1, *}$ Gang Yang $\odot,{ }^{2,3,4,5, \dagger}$ and Siyuan Zhang ${ }^{2,3, *}$ \\ ${ }^{1}$ School of Physics, Peking University, Beijing 100871, China \\ ${ }^{2}$ CAS Key Laboratory of Theoretical Physics, Institute of Theoretical Physics, Chinese Academy of Sciences, Beijing, 100190, China \\ ${ }^{3}$ School of Physical Sciences, University of Chinese Academy of Sciences, Beijing 100049, China \\ ${ }^{4}$ School of Fundamental Physics and Mathematical Sciences, Hangzhou Institute for Advanced Study, UCAS, Hangzhou 310024, China \\ ${ }^{5}$ International Centre for Theoretical Physics Asia-Pacific (Beijing/Hangzhou), UCAS, Beijing 100190, China
}

(Received 26 July 2021; accepted 30 September 2021; published 21 October 2021)

\begin{abstract}
We obtain full-color three-loop, three-point form factors of the stress-tensor supermultiplet and also of a length-3 half-Bogomol'nyi-Prasad-Sommerfield operator in $\mathcal{N}=4$ supersymmetric Yang-Mills (SYM) theory based on the color-kinematics duality and on-shell unitarity. The integrand results are verified by all planar and nonplanar unitarity cuts, and they satisfy the minimal power counting of loop momenta and diagrammatic symmetries. Interestingly, these three-loop solutions, while manifesting all dual Jacobi relations, contain a large number of free parameters; in particular, there are 24 free parameters for the form factor of the stress-tensor supermultiplet. Such degrees of freedom are due to a new type of generalized gauge transformation associated with the operator insertion for form factors. We also perform numerical integration and obtain consistent full-color infrared divergences and the known planar remainder. The form factors we obtain can be understood as the $\mathcal{N}=4$ SYM counterparts of three-loop Higgs plus three-gluon amplitudes in QCD and are expected to provide the maximally transcendental parts of the latter.
\end{abstract}

DOI: 10.1103/PhysRevLett.127.171602

Introduction.-Remarkable progress in our understanding of fundamental interactions has been made in both theoretical and experimental aspects in the last decades. One central impetus for these developments is the uncovering of surprising and intriguing mathematical structures hidden in microscopic scattering processes, where the maximally supersymmetric Yang-Mills theory $(\mathcal{N}=4$ SYM) has been an ideal laboratory. In the planar limit, the amplitudes are relatively well understood and even certain all-loop integrand constructions [1,2] and nonperturbative functional results $[3,4]$ have been achieved, thanks to the underlying integrability of planar $\mathcal{N}=4$ SYM, see [5] for a review. However, while going beyond the planar limit, the inclusion of color degrees of freedom complicates the problem, because the latter breaks many of the planar symmetries. As a result, understanding the nonplanar sector of the theory remains a very challenging task.

Taking advantage of the color "complications," a remarkable duality between color and kinematics was discovered by Bern et al. [6,7]. The color-kinematics (CK) duality

Published by the American Physical Society under the terms of the Creative Commons Attribution 4.0 International license. Further distribution of this work must maintain attribution to the author(s) and the published article's title, journal citation, and DOI. Funded by SCOAP. proposes that the gauge theory amplitudes can be organized in terms of trivalent graphs such that the kinematic numerators satisfy identities in one-to-one correspondence with color Jacobi identities. When combined together with the generalized unitarity method [8-10], this duality makes possible high-loop constructions of gauge amplitudes with full-color dependence, see, e.g., high-loop amplitudes in SYM [11-17] and pure YM theory [18-21], and also Sudakov form factors up to five loops in $\mathcal{N}=4 \mathrm{SYM}$ [22-24]. Apart from the significance to gauge theories, the duality also builds a bridge connecting gauge and gravity theories: the gravity amplitudes can be directly constructed from the Yang-Mills amplitudes in the CK-dual representation, via the "double copy" [7,25]. The so-called doublecopy property has many impressive utilities, for example, for understanding the ultraviolet properties of gravity theories [12,16,26-31]. A recent and extensive review of the duality and its applications can be found in [32].

The CK duality has been proved at tree level using string or gauge theory methods [33-35]. However, at loop level, the duality is still a conjecture and has only been shown by explicit constructions. Thus, it is very important to explore more examples and see to what extent the duality applies. It is worth pointing out that a loop representation fully manifesting CK duality is generally nontrivial to reach. For example, it has proven difficult to find such a representation for the five-loop, four-point amplitude in $\mathcal{N}=4$ SYM [16,29]. Another example is the all-plus 
two-loop, five-gluon amplitudes in pure YM theory: numerators with 12 powers of loop momenta that are much more than that of Feynman diagrams have to be used to realize the duality [21].

In this Letter, we obtain new three-loop solutions which manifest the color-kinematics duality for a class of threepoint form factors in $\mathcal{N}=4 \mathrm{SYM}$. Interestingly, the results contain a large number of free "gauge" parameters; for example, for the form factor of the stress-tensor supermultiplet, there are 24 free parameters. We would like to emphasize that our results belong to the "simplest" type of solutions, in the sense that they maintain all diagrammatic symmetries and they satisfy the minimal power-counting behavior expected in $\mathcal{N}=4 \mathrm{SYM}$. As we will discuss later, these free parameters originate from a new type of generalized gauge transformation, which is induced by the operator insertion in form factors.

Concretely, the form factors considered here describe the interaction between three on-shell states $\Phi_{i}$ and a gaugeinvariant operator $\mathcal{O}$ (see [36] for an introduction),

$$
\mathcal{F}_{\mathcal{O}}(1,2,3 ; q)=\int d^{D} x e^{-i q \cdot x}\left\langle\Phi_{1} \Phi_{2} \Phi_{3}|\mathcal{O}(x)| 0\right\rangle .
$$

Here we consider half-Bogomol'nyi-Prasad-Sommerfield (BPS) operators $\mathcal{O}_{L}=\operatorname{tr}\left(\phi^{L}\right)$, with $L=2$ and 3; in particular, $\mathcal{O}_{2}$ is a component of the stress-tensor supermultiplet. Besides the theoretical significance to $\mathrm{CK}$ duality, these form factors also bear phenomenological interest, due to their close relation to the Higgs-plus-threegluon amplitudes in QCD. In particular, both these two $\mathcal{N}=4$ form factors were found to coincide with the maximally transcendental part (i.e., functionally the most complicated part) of the corresponding QCD results up to two-loop order [37-41], providing examples of the general principle of maximal transcendentality [42,43]. As for higher-loop orders, the planar three-point form factor of $\mathcal{O}_{2}$ has been computed via bootstrap recently up to five loops [44] using the input from the form factor operator product expansion (OPE) $[45,46]$. Our result provides for the first time the three-loop nonplanar correction, for which powerful methods such as the OPE bootstrap are not yet applicable. For the form factor of $\mathcal{O}_{3}$, the two-loop result was given in [39], and here we provide the new three-loop result.

Below, we first give a brief review of CK duality and introduce our computational strategy. Then we explain our construction of CK-dual solutions. We further perform numerical integration, check the full-color infrared (IR) subtraction and obtain finite remainders. Finally, we discuss the interpretation of free parameters. The complete CK-dual solutions are given in the Supplemental Material [47].

Review and strategy.-An instructive example to illustrate the color-kinematics duality is the four-gluon tree
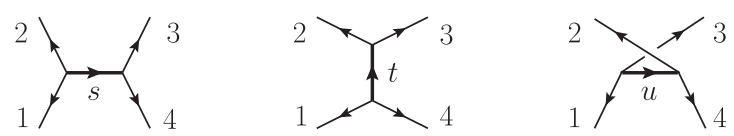

FIG. 1. Trivalent graphs for four-point tree amplitudes.

amplitude. It is possible to represent the amplitude in terms of three cubic graphs shown in Fig. 1,

$$
\boldsymbol{A}_{4}^{(0)}=\frac{C_{s} N_{s}}{s}+\frac{C_{t} N_{t}}{t}+\frac{C_{u} N_{u}}{u},
$$

where $C_{i}$ are color factors given as products of structure constants $\tilde{f}^{a b c}$ corresponding to each trivalent vertex, and $N_{i}$ are kinematic numerators that contain intrinsic physical information. Here we use the normalization $\operatorname{tr}\left(T^{a} T^{b}\right)=\delta^{a b}$ and $\tilde{f}^{a b c}=\operatorname{tr}\left(T^{a}\left[T^{b}, T^{c}\right]\right)$. The CK duality requires that the numerators should satisfy the dual Jacobi relations parallel to the corresponding color Jacobi relations as [6]

$$
C_{s}=C_{t}+C_{u} \Rightarrow N_{s}=N_{t}+N_{u} .
$$

While the CK duality has been proved at tree level, the striking point is that the duality can be generalized to loop level. Consider trivalent loop diagrams for form factors shown in Fig. 2: every internal propagator (not directly connected to the $q$ leg) is associated with a four-point tree subgraph. The three diagrams in Fig. 2 containing $s-, t$, and $u$-channel subgraphs are related by a color Jacobi relation, and $\mathrm{CK}$ duality requires that their numerators satisfy the dual Jacobi relation as

$$
N_{s}(l)=N_{t}(l)+N_{u}(l) .
$$

Note that the four-point subdiagrams in Fig. 2 have four offshell legs $l_{i}, i=a, b, c, d$, thus it is a highly nontrivial generalization from (3) to (4). These dual Jacobi relations will play a central role in the following three-loop constructions.

The general strategy of our constructions involves two major steps. The first step is to construct an ansatz of the loop integrand corresponding to a set of cubic graphs. By imposing the dual Jacobi relations (4), the numerators of different topologies are interlocked with each other, and an ansatz for the CK-dual integrand can be obtained efficiently. In the second step, we solve the ansatz by imposing physical constraints, where the main tool is the generalized unitarity method [8-10]. Below we implement the above

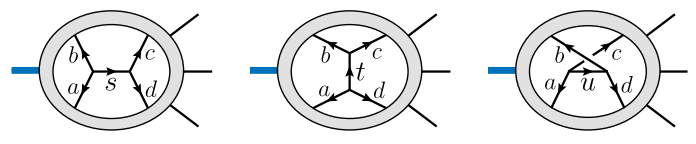

FIG. 2. Loop diagrams related by Jacobi relation. 


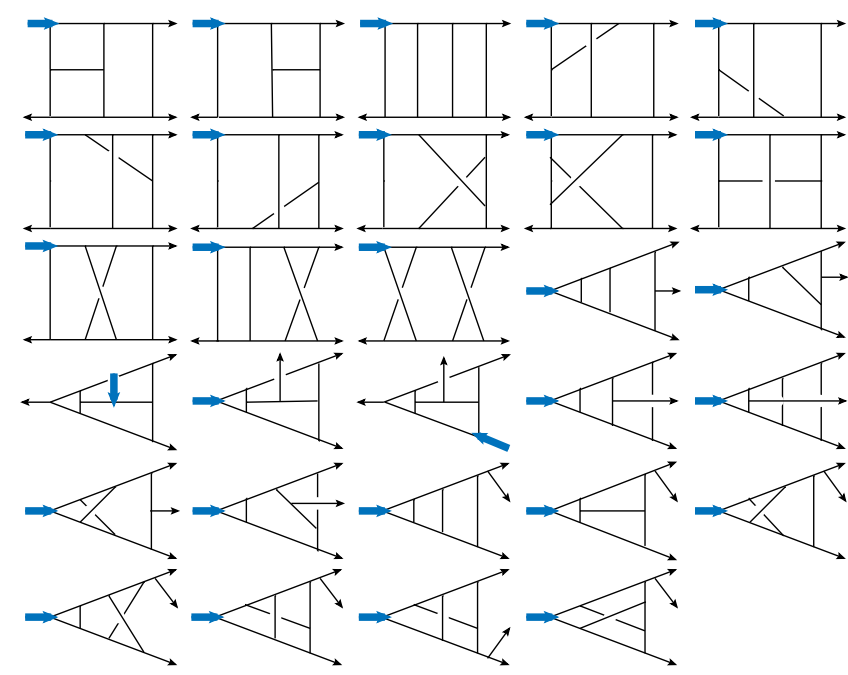

FIG. 3. Trivalent topologies for the form factor of $\operatorname{tr}\left(\phi^{2}\right)$.

strategy to construct the three-loop form factor solutions. Readers are also referred to $[12,36,48]$ for further details of general constructions.

Constructing CK-dual solutions.-We first discuss the form factor of $\operatorname{tr}\left(\phi^{2}\right)$. The starting point is to construct a set of trivalent graphs for the three-loop integrand. Each diagram contains four external legs: three on-shell legs $p_{i}$ and one off-shell leg $q$ associated with the operator. Following the empirical experience from the known highloop CK-dual solutions [12,22,23], we exclude graphs with tadpole, bubble, and triangle subgraphs, unless the triangle is attached to the $q$ leg. We find that there are 29 trivalent topologies to consider, as shown in Fig. 3.

Next we consider Jacobi relations for all four-point subgraphs of these topologies. It turns out that all topologies can be generated by two planar topologies, shown in Fig. 4, which are called master graphs. Once knowing the numerators of these two master graphs, all other numerators can be deduced via dual Jacobi relations (4).

To construct an ansatz for two planar master numerators, it is convenient to use zone variables $x_{i}$ [49], such as $x_{a 1}^{2}=$ $\left(x_{a}-x_{1}\right)^{2}=l_{a}^{2}$ shown in Fig. 4. Based on the nice UV properties of $\mathcal{N}=4$ SYM and half-BPS operators, we impose the minimal power-counting condition for loop momenta: a one-loop, $n$-point subgraph carries no more than $n-4$ powers of the corresponding loop momentum [12], with an exception that if the subgraph is a one-loop form factor, the maximal power is $n-3$ [22]. Specifically, for $N_{1}$ (the first master in Fig. 4), $x_{a}, x_{c}$ can appear at most
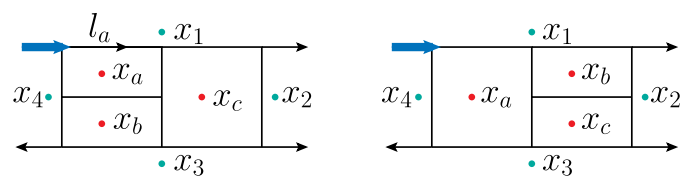

FIG. 4. Master graphs for $\operatorname{tr}\left(\phi^{2}\right)$ form factor.

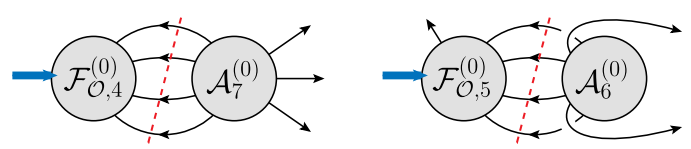

FIG. 5. Quadruple cuts for three-loop, three-point form factors.

once, so $\left(x_{a c}^{2}\right)^{1}$ or $\left(x_{a i}^{2}\right)^{1}\left(x_{c i}^{2}\right)^{1}$, with $i=1,2,3,4$, are allowed; whereas terms containing $x_{b}$ or containing more than one $x_{a}$ or $x_{c}$, such as $\left(x_{a c}^{2}\right)^{2},\left(x_{a 1}^{2}\right)^{2}$, are forbidden. For the other master numerator $N_{2}$, only $x_{a}$ can appear with maximal power 2, thus only $\left(x_{a i}^{2}\right)^{n}$ with power $n=2,1,0$ can appear. With these constraints, we obtain an ansatz form as linear combinations of monomials of zone variables with an overall dimension $\left[x^{8}\right]$, and two master numerators have 201 and 115 free parameters, respectively. Then we get an ansatz of the complete integrand with 316 parameters.

Given the ansatz, we further apply various constraints to fix the parameters. We first require numerators to respect the automorphism symmetries of corresponding graphs, see, e.g., [12,22]. This provides substantial constraints on the ansatz and reduces the number of parameters to 105 . To fix the remaining parameters, we apply (generalized) unitarity cuts [8-10], where two examples of the most complicated quadruple cuts are illustrated in Fig. 5. Interestingly, after imposing a spanning set of unitarity cuts, there are still 24 parameters left. We point out that our integrand correctly reproduce not only planar but also nonplanar cuts, which ensures that the unitarity constraints are complete. Finally, we check that all dual Jacobi relations are satisfied. Thus, we get the physical three-loop integrand solution with 24 free parameters that also manifests CK duality.

Following the above procedure, we also construct the three-loop integrand for the form factor of $\operatorname{tr}\left(\phi^{3}\right)$. Since the operator contains three scalars, in the trivalent diagrams the $q$ leg must be associated with a four-point vertex, as shown in Fig. 6. In this case, one can divide the three-loop topologies into two classes. The first class consists of

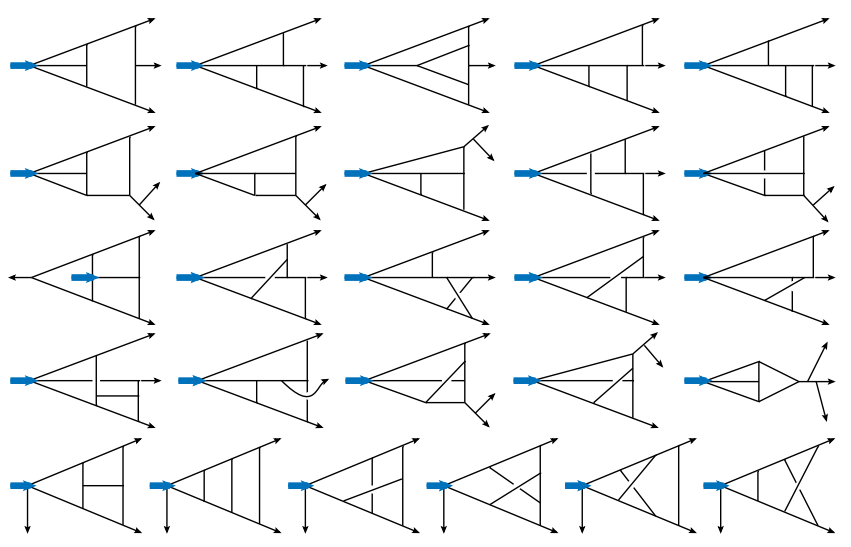

FIG. 6. Trivalent topologies for the form factor of $\operatorname{tr}\left(\phi^{3}\right)$. 


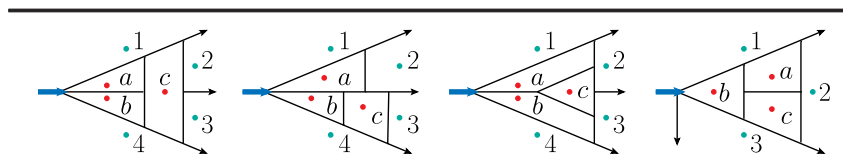

FIG. 7. Master graphs for $\operatorname{tr}\left(\phi^{3}\right)$ form factor.

integrals that involve all three external on-shell legs in the interactions, as shown in the first four rows in Fig. 6. In the second class, depicted by the diagrams in the last row of Fig. 6, one of the external on-shell legs is connected directly to the $q$ leg; these diagrams correspond to single-scale integrals. The dual Jacobi relations are decoupled between these two classes; consequently, the master topologies should be selected separately, as presented in Fig. 7: the first three planar masters are for the first class, and the last one is for the second class.

Since the procedure of constructing and solving the ansatz is similar to the $\operatorname{tr}\left(\phi^{2}\right)$ case, we will not go into details and only give a brief summary for the $\operatorname{tr}\left(\phi^{3}\right)$ case. An ansatz satisfying minimal power counting for the four master numerators has in total 295 parameters. After imposing the symmetry and unitarity constraints, we get the CK-dual integrand solution with ten free parameters.

For both form factors, we give solutions of master numerators in the Supplemental Material [47].

Results and checks.-The final CK-dual integrands of three-loop form factors can be summarized as

$$
\boldsymbol{F}_{\mathcal{O}_{L}, 3}^{(3)}=\mathcal{F}_{\mathcal{O}_{L}, 3}^{(0)} \sum_{\sigma_{3}} \sum_{i} \int \prod_{j=1}^{3} d^{D} \ell_{j} \frac{1}{S_{i}} \sigma_{3} \frac{C_{i} N_{i}}{\prod_{\alpha_{i}} P_{\alpha_{i}}^{2}},
$$

where $S_{i}$ are symmetry factors that remove the overcounting from the automorphism symmetries of the graphs, and $\sigma_{3}$ are the permutations of external on-shell momenta $p_{i}, i=1,2,3$ [50]. Explicit expressions of the symmetry factors $S_{i}$, color factors $C_{i}$, propagator lists $P_{\alpha_{i}}$, and numerators $N_{i}$ are given in the Supplemental Material [47].

The physical form factors constructed via the unitarity method are expected to be independent of the remaining free parameters. It turns out that all parameters cancel simply at the integrand level, and such a cancellation is related to the generalized gauge transformations induced by the operator insertion. We will come back to this in the discussion section. The simplified integrands of form factors in $N_{c}$ expansion can be given as

$$
\begin{gathered}
\boldsymbol{F}_{\mathcal{O}_{2}, 3}^{(3)}=\mathcal{F}_{\mathcal{O}_{2}, 3}^{(0)} \tilde{f}^{a_{1} a_{2} a_{3}}\left(N_{c}^{3} \mathcal{I}_{\mathcal{O}_{2}}^{(3)}+12 N_{c} \mathcal{I}_{\mathcal{O}_{2}, \mathrm{NP}}^{(3)}\right), \\
\boldsymbol{F}_{\mathcal{O}_{3}, 3}^{(3)}=\mathcal{F}_{\mathcal{O}_{3}, 3}^{(0)} \tilde{d}^{a_{1} a_{2} a_{3}} N_{c}^{3} \mathcal{I}_{\mathcal{O}_{3}}^{(3)},
\end{gathered}
$$

where $\tilde{d}^{a_{1} a_{2} a_{3}}=\operatorname{tr}\left(T^{a_{1}} T^{a_{2}} T^{a_{3}}\right)+\operatorname{tr}\left(T^{a_{1}} T^{a_{3}} T^{a_{2}}\right)$.

We see that the form factor of $\operatorname{tr}\left(\phi^{2}\right)$ contains a nonplanar three-loop correction. Notably, nondipole corrections of IR structures appear for the first time at three loops
[51]. To compare with these structures, we take a numerical approach to calculate the contributed three-loop integrals, using packages FIESTA [52] and pysecDec [53]. The evaluation of the nonplanar parts of these integrals turns out to be highly involved. To overcome this difficulty, we managed to organize the integrand into uniformly transcendental integrals to the necessary extent, which significantly improves the efficiency of computation; such a simplification has also been observed in the four-loop Sudakov form factor computation [54,55]. Our results give consistent IR divergences, for both the planar [56] and nonplanar parts [51,57]. Moreover, the three-loop planar remainder for the form factor of $\operatorname{tr}\left(\phi^{2}\right)$ confirms the recent bootstrap computation [44] (using also data from [58,59]). All these provide strong consistency checks of our results. Some details of the numerical checks are provided in the Supplemental Material [47]. More details on the simplification of the integrand and numerical computations will be given in [60].

Discussion.-An interesting finding of this Letter is that the integrand solutions contain a large number of free parameters, while at the same time manifesting all dual Jacobi relations. Practically, this is a very appealing property for the high-loop construction using the CK-dual ansatz. Such free parameters can be understood as deformations of the integrand that do not change the final form factor result. Below, we discuss the origin of these deformations.

We first point out that the free parameters we find have no relation to the traditional gauge transformations that correspond to changing external polarization vectors as $\varepsilon_{i}^{\mu} \rightarrow \varepsilon_{i}^{\mu}+\alpha p_{i}^{\mu}$ for arbitrary $\alpha$. This is simply because the loop corrections are independent of polarization vectors.

Another type of integrand transformation is the so-called generalized gauge transformation (GGT) [7]. For example, one can deform the numerators associated with $s^{-}, t-$, and $u$-channel trivalent graphs as

$N_{s} \rightarrow N_{s}+s \Delta, \quad N_{t} \rightarrow N_{t}-t \Delta, \quad N_{u} \rightarrow N_{u}-u \Delta$,

for arbitrary $\Delta$ without changing the amplitude or form factor results. At loop level, such a transformation typically breaks the dual Jacobi relations, since $s+t+u \neq 0$, see Fig. 2.

For form factors, a new type of generalized gauge transformations appears, due to the insertion of local operators. To illustrate this point, let us consider the simpler two-loop, three-point form factor of $\operatorname{tr}\left(\phi^{2}\right)$. In this case, we find that the two-loop CK-dual representation (with minimal power counting) also contains four free parameters. Consider the graphs in Fig. 8: the first two topologies share the same color factor 


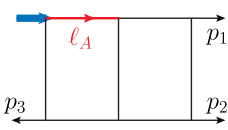

(a)

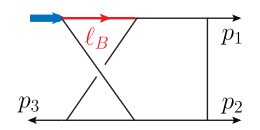

(b)

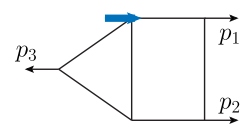

(c)
FIG. 8. Graph examples of two-loop form factor of $\operatorname{tr}\left(\phi^{2}\right)$. (a), (b) Have the same color factor and, after shrinking the propagators labeled by $\ell_{A}$ and $\ell_{B}$, they both reduce to (c).

$$
C_{a}=C_{b},
$$

since the color factor of the local operator $\operatorname{tr}\left(\phi^{2}\right)$ is a $\delta$ function in color space. This implies that one can make a deformation of the corresponding numerators as

$$
N_{a} \rightarrow N_{a}+\ell_{A}^{2} \Delta, \quad N_{b} \rightarrow N_{b}-\ell_{B}^{2} \Delta
$$

which leaves the full integrand unchanged,

$$
C_{a} I_{a}\left[\ell_{A}^{2} \Delta\right]-C_{b} I_{b}\left[\ell_{B}^{2} \Delta\right]=\left(C_{a}-C_{b}\right) I_{c}[\Delta]=0 .
$$

Here $I_{a, b, c}$ are integrals related to the topologies in Fig. 8. Note that (10) has different color-algebraic origin from (8). To distinguish these two transformations, we call (10) the "operator-induced" GGT, while referring to (8) as the "Jacobi-induced" GGT.

The high-dimensional solution space in the CK-dual representation is closely related to the operator-induced GGT for form factors. Indeed, in order to show that the integrands in the solution space (with different choices of the free parameters) are equivalent, one must apply the color relations (9) and the operator-induced GGT for the considered form factors. Explicit two-loop examples are given in the Supplemental Material [47].

Finally, we recall that gravity amplitudes can be obtained through a double copy of gauge amplitudes in the CK-dual representation, where the Jacobi-induced GGT leads to diffeomorphism invariance in gravity [32]. Given the CKdual solutions for form factors, one may ask if there is a physical meaning of performing a double copy in this case. In order to have a consistent double-copy result, where color factors $C_{i}$ are replaced by kinematic numerators $\tilde{N}_{i}$,

$$
\sum_{i} \frac{C_{i} N_{i}}{\prod D_{i, \alpha}} \Rightarrow \sum_{i} \frac{\tilde{N}_{i} N_{i}}{\prod D_{i, \alpha}}
$$

the $\tilde{N}_{i}$ should satisfy all corresponding color relations, including those operator-induced color relations such as in the case of Fig. 8,

$$
C_{a}=C_{b} \Rightarrow \tilde{N}_{a}=\tilde{N}_{b}
$$

Otherwise, the free parameters in $N_{i}$ will not cancel in the double-copy result. We find that, for the two- and threeloop, three-point form factors, there is no numerator solution satisfying such a requirement. This implies that the operator-induced GGT may not give diffeomorphism invariance in gravity through double copy, which seems consistent with the known fact that local diffeomorphisminvariant operators do not exist in gravity. It would be interesting to explore this point further, since the argument here does not exclude the possibilities that the double-copy picture may apply to form factors in special choices of gauge, as well as their possible connections to certain nonlocal observables in gravity.

It is a pleasure to thank Yuchen Ding, Yuanhong Guo, Song He, and Yanqing Ma for interesting discussions. This work is supported in part by the National Natural Science Foundation of China (Grants No. 11822508, No. 11935013, and No. 12047503), and by the Key Research Program of the Chinese Academy of Sciences, Grant No. XDPB15. We also are thankful for the support of the HPC Cluster of ITP-CAS and CAS Xiandao-1 computing environment.

*linguandak@pku.edu.cn

†yangg@itp.ac.cn

*zhangsiyuan@itp.ac.cn

[1] N. Arkani-Hamed, J. L. Bourjaily, F. Cachazo, S. CaronHuot, and J. Trnka, J. High Energy Phys. 01 (2011) 041.

[2] N. Arkani-Hamed, J. L. Bourjaily, F. Cachazo, A. B. Goncharov, A. Postnikov, and J. Trnka, Grassmannian Geometry of Scattering Amplitudes (Cambridge University Press, Cambridge, England, 2016).

[3] B. Basso, A. Sever, and P. Vieira, Phys. Rev. Lett. 111, 091602 (2013).

[4] B. Basso, A. Sever, and P. Vieira, J. Phys. A 49, 41LT01 (2016).

[5] N. Beisert et al., Lett. Math. Phys. 99, 3 (2012).

[6] Z. Bern, J. J. M. Carrasco, and H. Johansson, Phys. Rev. D 78, 085011 (2008).

[7] Z. Bern, J. J. M. Carrasco, and H. Johansson, Phys. Rev. Lett. 105, 061602 (2010).

[8] Z. Bern, L. J. Dixon, D. C. Dunbar, and D. A. Kosower, Nucl. Phys. B425, 217 (1994).

[9] Z. Bern, L. J. Dixon, D. C. Dunbar, and D. A. Kosower, Nucl. Phys. B435, 59 (1995).

[10] R. Britto, F. Cachazo, and B. Feng, Nucl. Phys. B725, 275 (2005).

[11] J. J. M. Carrasco and H. Johansson, Phys. Rev. D 85, 025006 (2012).

[12] Z. Bern, J. J. M. Carrasco, L. J. Dixon, H. Johansson, and R. Roiban, Phys. Rev. D 85, 105014 (2012).

[13] Z. Bern, S. Davies, and T. Dennen, Phys. Rev. D 88, 065007 (2013).

[14] Z. Bern, S. Davies, and T. Dennen, Phys. Rev. D 90, 105011 (2014).

[15] H. Johansson, G. Klin, and G. Mogull, J. High Energy Phys. 09 (2017) 019.

[16] Z. Bern, J. J. M. Carrasco, W.-M. Chen, H. Johansson, R. Roiban, and M. Zeng, Phys. Rev. D 96, 126012 (2017). 
[17] G. Klin, G. Mogull, and A. Ochirov, J. High Energy Phys. 07 (2019) 120.

[18] R. H. Boels, R. S. Isermann, R. Monteiro, and D. O’Connell, J. High Energy Phys. 04 (2013) 107.

[19] Z. Bern, S. Davies, T. Dennen, Y.-t. Huang, and J. Nohle, Phys. Rev. D 92, 045041 (2015).

[20] Z. Bern, S. Davies, and J. Nohle, Phys. Rev. D 93, 105015 (2016).

[21] G. Mogull and D. O'Connell, J. High Energy Phys. 12 (2015) 135.

[22] R. H. Boels, B. A. Kniehl, O. V. Tarasov, and G. Yang, J. High Energy Phys. 02 (2013) 063.

[23] G. Yang, Phys. Rev. Lett. 117, 271602 (2016).

[24] G. Lin and G. Yang, J. High Energy Phys. 04 (2021) 176.

[25] Z. Bern, T. Dennen, Y.-t. Huang, and M. Kiermaier, Phys. Rev. D 82, 065003 (2010).

[26] Z. Bern, S. Davies, T. Dennen, and Y.-t. Huang, Phys. Rev. Lett. 108, 201301 (2012).

[27] Z. Bern, S. Davies, T. Dennen, and Y.-t. Huang, Phys. Rev. D 86, 105014 (2012).

[28] Z. Bern, S. Davies, T. Dennen, A. V. Smirnov, and V. A. Smirnov, Phys. Rev. Lett. 111, 231302 (2013).

[29] Z. Bern, J. J. Carrasco, W.-M. Chen, H. Johansson, and R. Roiban, Phys. Rev. Lett. 118, 181602 (2017).

[30] Z. Bern, J. J. Carrasco, W.-M. Chen, A. Edison, H. Johansson, J. Parra-Martinez, R. Roiban, and M. Zeng, Phys. Rev. D 98, 086021 (2018).

[31] E. Herrmann and J. Trnka, J. High Energy Phys. 02 (2019) 084.

[32] Z. Bern, J. J. Carrasco, M. Chiodaroli, H. Johansson, and R. Roiban, arXiv:1909.01358.

[33] N. E. J. Bjerrum-Bohr, P. H. Damgaard, and P. Vanhove, Phys. Rev. Lett. 103, 161602 (2009).

[34] S. Stieberger, arXiv:0907.2211.

[35] B. Feng, R. Huang, and Y. Jia, Phys. Lett. B 695, 350 (2011).

[36] G. Yang, Sci. China Phys. Mech. Astron. 63, 270001 (2020).

[37] A. Brandhuber, G. Travaglini, and G. Yang, J. High Energy Phys. 05 (2012) 082.

[38] T. Gehrmann, M. Jaquier, E. Glover, and A. Koukoutsakis, J. High Energy Phys. 02 (2012) 056.

[39] A. Brandhuber, B. Penante, G. Travaglini, and C. Wen, J. High Energy Phys. 08 (2014) 100.

[40] A. Brandhuber, M. Kostacinska, B. Penante, and G. Travaglini, Phys. Rev. Lett. 119, 161601 (2017).

[41] Q. Jin and G. Yang, Phys. Rev. Lett. 121, 101603 (2018).

[42] A. V. Kotikov and L. N. Lipatov, Nucl. Phys. B661, 19 (2003); B685, 405(E) (2004).
[43] A. Kotikov, L. Lipatov, A. Onishchenko, and V. Velizhanin, Phys. Lett. B 595, 521 (2004).

[44] L. J. Dixon, A. J. McLeod, and M. Wilhelm, J. High Energy Phys. 04 (2021) 147.

[45] A. Sever, A. G. Tumanov, and M. Wilhelm, Phys. Rev. Lett. 126, 031602 (2021).

[46] A. Sever, A. G. Tumanov, and M. Wilhelm, arXiv:2105 13367.

[47] See Supplemental Material at http://link.aps.org/ supplemental/10.1103/PhysRevLett.127.171602 for ancillary files of CK-dual solutions, as well as a supplemental pdf file on the numerical results and the two-loop example on free-parameter cancellations.

[48] J. J. M. Carrasco, in Proceedings of the Theoretical Advanced Study Institute (TASI 2014), Boulder, CO, 2014 (World Scientific, Singapore, 2015), https://doi.org/ 10.1142/9789814678766_0011.

[49] J. M. Drummond, J. Henn, V. A. Smirnov, and E. Sokatchev, J. High Energy Phys. 01 (2007) 064.

[50] The permutation operator $\sigma_{3}$ acts on $N_{i}, P_{\alpha_{i}}^{2}$, and $C_{i}$ and changes external momenta and color indices associated with them, respectively. For the form factor of $\operatorname{tr}\left(\phi^{2}\right)$, acting $\sigma_{3}$ on $N_{i}$ may give an extra sign, i.e., $\sigma_{3}\left[N_{i}\left(p_{1}, p_{2}, p_{3}\right)\right]=$ $\operatorname{sign}\left(\sigma_{3}\right) N_{i}\left(\sigma_{3}\left(p_{1}\right), \sigma_{3}\left(p_{2}\right), \sigma_{3}\left(p_{3}\right)\right)$, since the tree-level form factor [factorized as $\mathcal{F}^{(0)}$ ] gives a $\operatorname{sign}\left(\sigma_{3}\right)$ when permuting external lines.

[51] E. Gardi, Ø. Almelid, and C. Duhr, Proc. Sci., LL2016 (2016) 058.

[52] A. V. Smirnov, Comput. Phys. Commun. 204, 189 (2016).

[53] S. Borowka, G. Heinrich, S. Jahn, S. P. Jones, M. Kerner, J. Schlenk, and T. Zirke, Comput. Phys. Commun. 222, 313 (2018).

[54] R. H. Boels, T. Huber, and G. Yang, Phys. Rev. Lett. 119, 201601 (2017).

[55] R. H. Boels, T. Huber, and G. Yang, J. High Energy Phys. 01 (2018) 153.

[56] Z. Bern, L. J. Dixon, and V. A. Smirnov, Phys. Rev. D 72, 085001 (2005).

[57] J. M. Henn and B. Mistlberger, Phys. Rev. Lett. 117, 171601 (2016).

[58] M. Spradlin, A. Volovich, and C. Wen, Phys. Rev. D 78, 085025 (2008).

[59] T. Gehrmann, J. M. Henn, and T. Huber, J. High Energy Phys. 03 (2012) 101.

[60] G. Lin, G. Yang, and S. Zhang (to be published). 